\title{
Multiple-Pulse Operation and Bound States of Solitons in Passive Mode-Locked Fiber Lasers
}

\author{
A. Komarov, ${ }^{1}$ F. Amrani, ${ }^{2}$ A. Dmitriev, ${ }^{1}$ K. Komarov, ${ }^{3}$ D. Meshcheriakov, ${ }^{1,3}$ and F. Sanchez ${ }^{2}$ \\ ${ }^{1}$ Department of Laser Systems, Novosibirsk State Technical University, 630092 Novosibirsk, Russia \\ ${ }^{2}$ Laboratoire de Photonique d'Angers EA 4644, Université d'Angers, 49000 Angers, France \\ ${ }^{3}$ Institute of Automation and Electrometry, Russian Academy of Sciences, 630090 Novosibirsk, Russia \\ Correspondence should be addressed to A. Komarov, andnsk@iae.nsk.su
}

Received 3 May 2011; Accepted 25 July 2011

Academic Editor: Sonia Boscolo

Copyright (๑) 2012 A. Komarov et al. This is an open access article distributed under the Creative Commons Attribution License, which permits unrestricted use, distribution, and reproduction in any medium, provided the original work is properly cited.

We present results of our research on a multiple-pulse operation of passive mode-locked fiber lasers. The research has been performed on basis of numerical simulation. Multihysteresis dependence of both an intracavity energy and peak intensities of intracavity ultrashort pulses on pump power is found. It is shown that the change of a number of ultrashort pulses in a laser cavity can be realized by hard as well as soft regimes of an excitation and an annihilation of new solitons. Bound steady states of interacting solitons are studied for various mechanisms of nonlinear losses shaping ultrashort pulses. Possibility of coding of information on basis of soliton trains with various bonds between neighboring pulses is discussed. The role of dispersive wave emitted by solitons because of lumped intracavity elements in a formation of powerful soliton wings is analyzed. It is found that such powerful wings result in large bounding energies of interacting solitons in steady states. Various problems of a soliton interaction in passive mode-locked fiber lasers are discussed.

\section{Introduction}

Lasers generating ultrashort optical pulses are widely employed in diversified areas of science, technology, and engineering [1-7]. Applications of such lasers range from testing of ultrahigh speed semiconductor devices to precision processing of materials, from triggering of tracing chemical reactions to sophisticated surgical applications in medicine. These lasers are used for study of ultrahigh speed processes in atomic and molecular physics, in solid-state physics, and in chemistry and biology. They are employed for investigation of light-matter interactions under ultrahigh intensity levels. Lasers of ultrashort optical pulse with a high repetition rate are a key element in high-speed optical communications. Ultrashort pulse lasers are extensively used for micromachining, biomedical diagnostic, in light detection, and ranging (lidar) systems, and so forth.

The great diversity of applications of ultrashort pulse lasers calls for further development and perfection of this type of quantum generators. At the present time, one of main ways for creation of perfect ultrashort pulse sources is related to passive mode-locked fiber lasers [8-16]. Nonlinear losses forming ultrashort pulses in fiber lasers are usually realized by the nonlinear polarization rotation technique. These lasers have unique potentialities. They are reliable, compact, flexible, and of low cost. Such generators can be conveniently pumped with commercially available semiconductor lasers. The nonlinear losses based on the nonlinear polarization rotation technique are fast, practically inertia-free. For them, the depth of the modulation and the saturating intensity are easily controlled through the orientation angles of intracavity phase plates. The great variety of operating regimes is an important feature of this type of lasers. Indeed, these lasers have demonstrated bistability between continuous wave and mode-locking regimes, spike operation, and Qswitching $[17,18]$. They can operate either with a single pulse in the laser cavity or in a multiple-pulse regime. The latter is connected with the effect of a quantization of intracavity lasing radiation into individual identical solitons [17-21]. Lasers operating in multiple-pulse regimes demonstrate multistability: the number of pulses in an established operation depends on initial conditions $[17,20]$. 
The dependence of the number of pulses on pumping and on orientation angles exhibits hysteresis phenomena [22]. Analogical regimes are realized in lasers with another mechanisms of nonlinear losses (semiconductor saturable absorber mirror (SESAM), saturable absorbers based on quantum dots, carbon nanotubes, graphene, and so on [23-25]).

The type of a soliton interaction plays a crucial role in the established multiple-pulse regimes of fiber lasers [26-30]. In the case of pulse attraction, bound solitons structures can be formed. Such structures were theoretically and experimentally investigated by many authors $[11,13,16$, $26,28,30-32]$. Possibility of a realization of strong bonds between solitons ( $\sim 10 \%$ of an individual soliton energy) was found in the paper [33]. As this takes place, steady states of pair interacting solitons form a two-soliton molecule with a set of energy levels corresponding to various types of bonds between pulses. With a use of this effect, the highstable noise-proof information sequences of bound solitons can be realized. In such sequences, a high-density coding of the information is realized through various distributions of different energy bonds along the soliton chains.

A long-distance mechanism of repulsion of ultrasort pulses results in the regime of harmonic passive modelocking [34-37] (the regime of a multiple-pulse generation in which distances between all neighboring pulses take the same value). The harmonic passive mode-locked fiber lasers are of great interest as ultrashort optical pulse sources with a high repetition rate which are employed in high-speed optical communications. This lasing regime can be also realized on basis of a sequence of bound solitons with a single type of a bond between neighboring pulses which fills completely a total laser resonator. In this case, the expected rate of repetition of ultrashort pulses in the output laser radiation is of the order of inverse ultrashort pulse duration and can lay in the terahertz frequency range for subpicosecond pulses $[38,39]$.

A quantization of intracavity radiation into individual identical solitons is a useful phenomenon for a creation of ultrashort pulse generators with a high rate of a repetition of ultrashort pulses. The greater number of pulses in laser cavity results in the greater rate of the repetition of pulses in output radiation. However, this phenomenon is a serious obstacle for creation of generators with high energy of individual pulses. Really, in consequence of this phenomenon, an increase of pumping results in an increase in number of pulses in the laser resonator, thus the energy of an individual pulse remains approximately as before.

The effective control of intersoliton interactions opens new opportunities for management of generation regimes of fiber lasers. For realization of such control, it is necessary to know the properties of soliton interaction at a fundamental level. In this paper, we present our results on a formation of multiple-pulse regimes connected with interaction of lasing solitons through a gain medium, inertia-free nonlinear losses, and a nonlinear refractive index. The paper contains the original results on the multihysteresis dependence of the lasing energy characteristics (a total energy of an intracavity radiation, peak intensities of individual solitons, an amplification coefficient, etc.) on pumping, the results

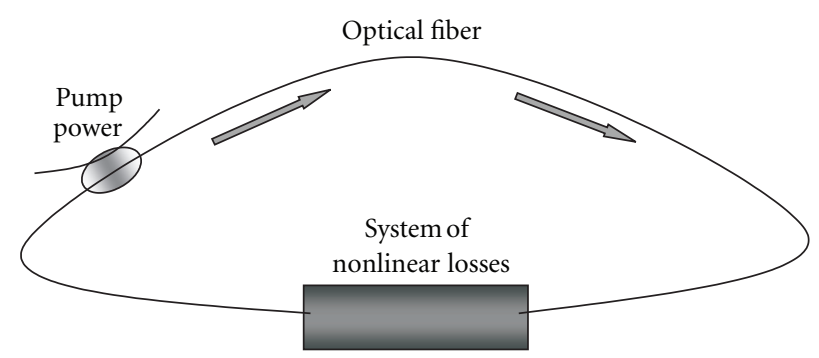

FIGURE 1: Schematic representation of the investigated ring passive mode-locked fiber laser.

on the realization of hard and soft regimes of excitation and annihilation of solitons in a laser cavity under multiplepulse operation, and the results on an interaction of solitons through dispersive waves (these waves are emitted by solitons because of lumped elements in a laser cavity). For a completeness of a description of these phenomena, we also discuss earlier our results on the given subjects (a soliton quantization, information ultrashort pulse trains in fiber lasers with the nonlinear polarization rotation technique, etc.).

This paper is organized as follows. In Section 2, we present the results on multihysteresis phenomena due to a competition and a coexistence of solitons in a laser cavity. Section 3 is devoted to bound steady states related with the interaction of solitons through an interference of their wings in a intracavity medium with fast nonlinear losses and Kerr nonlinearity of refractive index. In Section 4, we analyze the interaction of solitons in fiber laser with lumped saturable absorber. Section 5 is devoted to mechanisms of a realization of powerful long-distance soliton wings which are connected with dispersive waves emitted by solitons because of lumped intracavity elements. Such powerful wings result in a strong intersoliton interaction with great bound energies. In Section 6, we discuss the presented results. The most important ones from them are presented in Section 7.

\section{Multiple-Pulse Operation and Hysteresis Phenomena in Fiber Lasers}

The laser under investigation is schematically represented in Figure 1. The system forming nonlinear losses through a nonlinear polarization rotation technique was described in detail in [20]. For isotropic fiber, it involves all necessary elements for the polarization control. In this system, a radiation passes sequentially through the first quarter wave plate, the second half wave plate, the polarizer, and the third quarter wave plate. The space orientation of the three phase plates is determined by the angles $\alpha_{1}, \alpha_{2}, \alpha_{3}$, respectively ( $\alpha_{i}$ is an angle of one eigenaxis of a corresponding plate with respect to the passing axis of the polarizer). After the polarizing isolator, the electric field has a well-defined linear polarization. Such state of polarization does not experience polarization rotation in the fiber because the rotation angle is proportional to the area of the polarization ellipse. Consequently, it is necessary to place the third 
quarter wave plate after the polarizer which transforms the linear polarization into the elliptic one. The rotation of the polarization ellipse resulting from the optical Kerr nonlinearity is proportional to the light intensity, the area of the polarization ellipse, and the fiber length. At the output of the fiber, the direction of the elliptical polarization of the central part of the pulse can be rotated towards the passing axis of the polarizer by the second half wave plate. Then, this elliptical polarization can be transformed into a linear one by the first quarter wave plate. In this situation, the losses for the central part of the pulse are minimized while the wings undergo strong losses. The evolution of the radiation in the investigated laser is described by the following set of equations [20]:

$$
\begin{aligned}
\frac{\partial E}{\partial \zeta}= & \left(\mathrm{D}_{\mathrm{r}}+i \mathrm{D}_{\mathrm{i}}\right) \frac{\partial^{2} E}{\partial \tau^{2}}+\left(G+i q|E|^{2}\right) E \\
E_{n+1(\tau)}=-\eta[ & \cos \left(p I_{n}+\alpha_{0}\right) \cos \left(\alpha_{1}-\alpha_{3}\right) \\
& \left.+i \sin \left(p I_{n}+\alpha_{0}\right) \sin \left(\alpha_{1}+\alpha_{3}\right)\right] E_{n}(\tau)
\end{aligned}
$$

where $\mathrm{E}(\zeta, \tau)$ is the electric field amplitude, $\tau$ is a time coordinate expressed in units $\delta t=\sqrt{\left|\beta_{2}\right| L / 2}$ (here $\beta_{2}$ is the second-order group-velocity dispersion for fiber and $L$ is the fiber length), $\zeta$ is the normalized propagation distance $\zeta=z / L, \mathrm{D}_{r}$ and $\mathrm{D}_{i}$ are the frequency dispersions for a gainloss and for a refractive index, respectively, and $q$ is the Kerr nonlinearity. The term $G$ in the second parenthesis in (1) describes the saturable amplification

$$
G=\frac{a}{1+b J}
$$

where $J=\int|E|^{2} d \tau$ is the total energy of the intracavity radiation (the integration is carried out on the whole roundtrip period), $a$ is the pumping parameter, and $b$ is the saturation one). The second term in these parentheses is connected with Kerr nonlinearity of the fiber. Equation (2) determines the relation between the time-distributions of the field before and after $n$-th pass of radiation through the polarizer ( $\eta$ is the transmission coefficient of the intracavity polarizer). Parameters $\alpha_{0}, I, p$ are determined by relations $\alpha_{0}=2 \alpha_{2}-\alpha_{1}-\alpha_{3}, I=|E|^{2}, p=\sin \left(2 \alpha_{3}\right) / 3$. The amplitude $E(\tau)$ is subject to periodic boundary conditions with period equal to one round trip.

The numerical procedure starts from the evaluation of the electric field after passing through the Kerr medium, the phase plates, and the polarizer, using (2). The resulting electric field is then used as the input field to solve (1) over a distance L, using a standard split-step Fourier algorithm. The computed output field is used as the new input for (2). This iterative procedure is repeated until a steady state is achieved. For the numerical simulations, we use typical parameters of ytterbium- and erbium-doped fiber lasers operating in the normal and anomalous dispersion regimes.

Figure 2 shows the distribution of radiation in a laser resonator $I(\tau)$ as the function of a number of passes of the field through the cavity $\zeta$. After a transient process, the

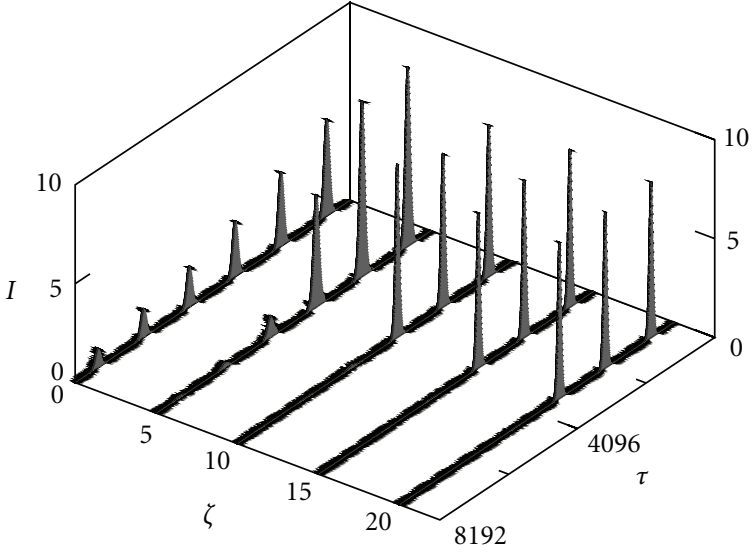

Figure 2: Transient evolution of the field in the fiber laser. The temporal distribution of the intracavity intensity $I(\tau)$ versus the round-trip number $\zeta . a=3, b=0.017, q=1, D_{i}=-1$, $D_{r}=D_{r 0} G, D_{r 0}=0.34, \alpha_{0}=-1.1, \alpha_{3}=-\alpha_{1}=0.2$.

stationary operation with identical pulses is realized. The difference in the peak intensities of initial pulses models a random spread in amplitudes of pulses of an initial noise radiation. Figure 3 demonstrates the number of identical pulses in a laser cavity in the established stationary operation as the function of pumping $a$. With changing pump $a$ the pulses are created and annihilated one by one. The corresponding changes in the intracavity radiation energy $J$, in the peak intensity of intracavity identical pulses $I_{0}$, and in the amplification $G$ are shown in Figures 4, 5, and 6 , respectively. The vertical arrows in these figures are connected to a change of a number of pulses $N$ in the laser resonator that is due to a change of pumping $a$ (see Figure 3). From the dependences $I_{0}=I_{0}(a)$ and $G=G(a)$ (see Figures 5 and 6), we obtain the dependence $G=G\left(I_{0}\right)$. The total amplification of a steady-state pulse consists of two parts. The first part is due to the gain $G\left(I_{0}\right)$. The second part $\Lambda$ is due to nonlinear losses and a dispersion of intracavity elements. In stationary regime, the total amplification is equal to zero, that is $\Lambda=-G\left(I_{0}\right)$. The dependence of nonlinear-dispersion part $\Lambda$ of the total amplification coefficient for an individual soliton as the function of its peak intensity $I_{0}$ is presented in Figure 7. This dependence is a key factor determining a competition and a coexistence of solitons in an established stationary regime. In the interval $I_{0}<I_{c r 2}$, the dependence $\Lambda=\Lambda\left(I_{0}\right)$ is monotonously increasing. Here, the pulse with a greater peak intensity $I_{0}$ has a greater amplification $\Lambda$. As a result of the soliton competition, no multiple-pulse regimes is realized. After a transient process, the generation with single soliton in a laser cavity is established. In the interval $I_{c r 2}<$ $I_{0}<I_{c r 1}$, a multiple-pulse generation is possible. Here, the pulse with a greater peak intensity $I_{0}$ has a less amplification $\Lambda$. As a result, after transient process, all pulses in a laser cavity have the same peak intensity $I_{0}$ and the same other parameters (a duration, an energy, a chirp, and so on). It is, so-called, the effect of quantization of a laser radiation on identical solitons. When, with decreasing pump $a$, the peak intensity of identical pulses $I_{0}$ reaches the value $I_{c r 2}$ 


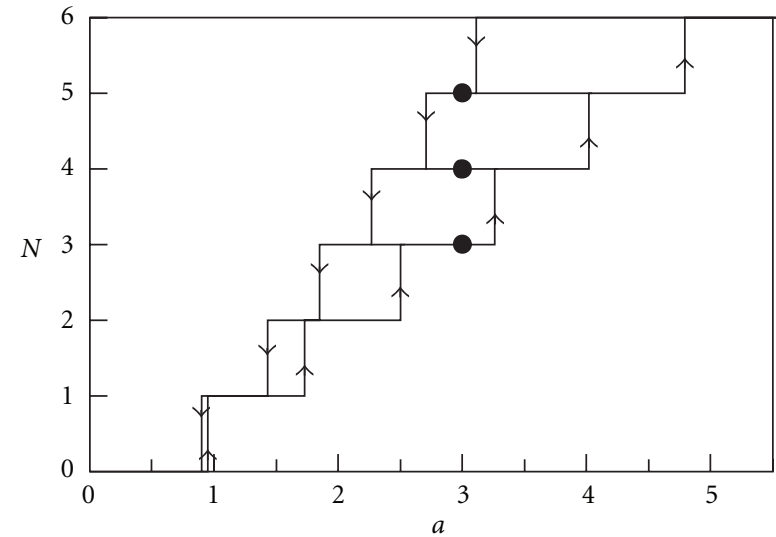

Figure 3: Multihysteresis dependence of the number of pulses $N$ in established stationary operation on pumping $a$.

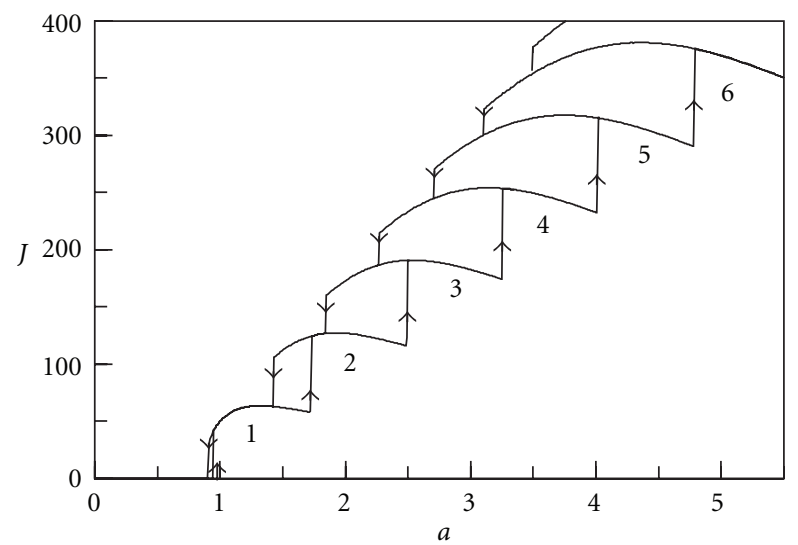

FIgURE 4: Dependence of the intracavity radiation energy $J$ in established operation on pumping $a$. Digits indicate a number of pulses in a laser cavity for a corresponding branch.

the pulses disappear one by one as one can see in Figure 3 (the left step dependence $N=N(a)$ ). To the contrary, when, with increasing pump, the peak intensity of identical pulses reaches the value $I_{c r 1}$, new pulses appear in the cavity one by one (the right step dependence in Figure 3). They are formed from weak seed pulses of a radiation noise. As a rule, the solitons appear and disappear by a threshold way (hard excitation and annihilation of the solitons [40]). That is, a peak intensity of each soliton is changed by a jump from 0 to $I_{0}$ and from $I_{0}$ to 0 (see Figure 5). However, the soft regime of excitation and annihilation of solitons [40] (a continuous change of the peak intensity with continuously changing pump $a$ ) is also possible. Figure 8 demonstrates the examples of the two types of changes in the second pulse (the first pulse already exists in the laser resonator). The corresponding dependences $\Lambda=\Lambda\left(I_{0}\right)$ are presented in Figure 9. The soft excitation and annihilation of pulses are realized if the slope of the decreasing part of the dependence $\Lambda=\Lambda\left(I_{0}\right)$ is greater than the slope of the increasing one.

It should be pointed out that the dependence $\Lambda=\Lambda\left(I_{0}\right)$ determines the phase relations of interacting solitons in bound steady states: for peak intensities in the vicinity of top

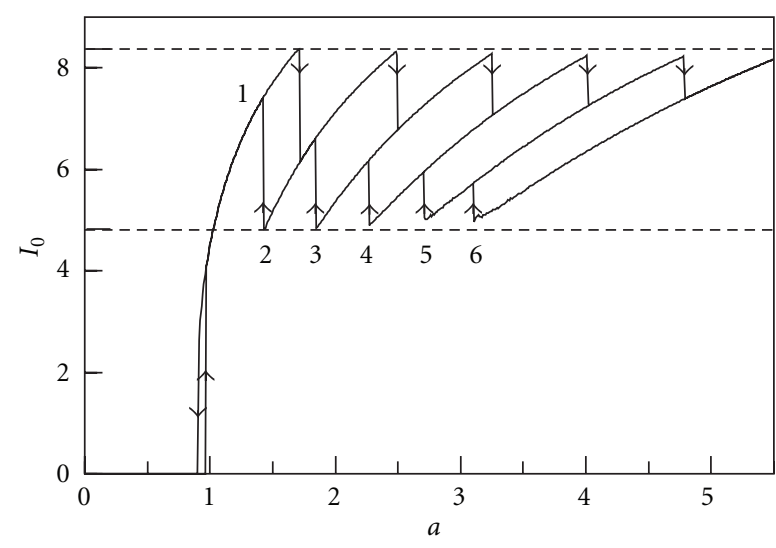

FIGURE 5: Dependence of the peak intensity $I_{0}$ of intracavity identical pulses in established operation on pumping $a$.

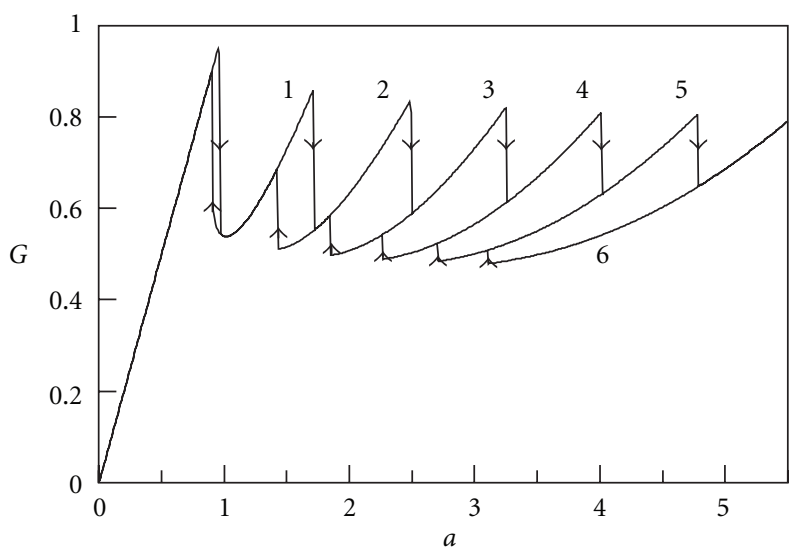

FIgURE 6: Dependence of the gain $G$ on pumping $a$.

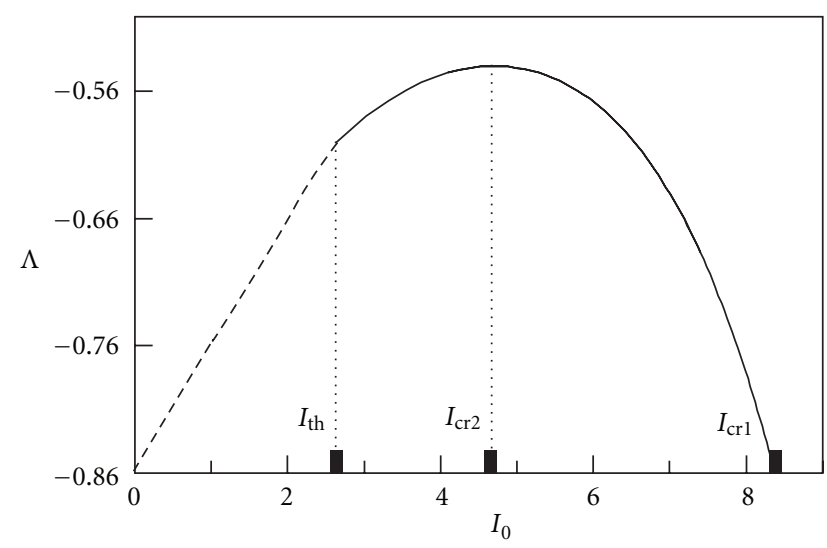

FIGURE 7: Dependence of the nonlinear-dispersion part of amplification for intracavity pulses $\Lambda$ on their peak intensity $I_{0}$. The fragment of an unstable single-pulse generation is shown as a dashed line. The value $I_{c r 2}$ corresponds maximum $\Lambda=\Lambda\left(I_{0}\right)$. $\Lambda\left(I_{c r 1}\right)=\Lambda(0)$. 


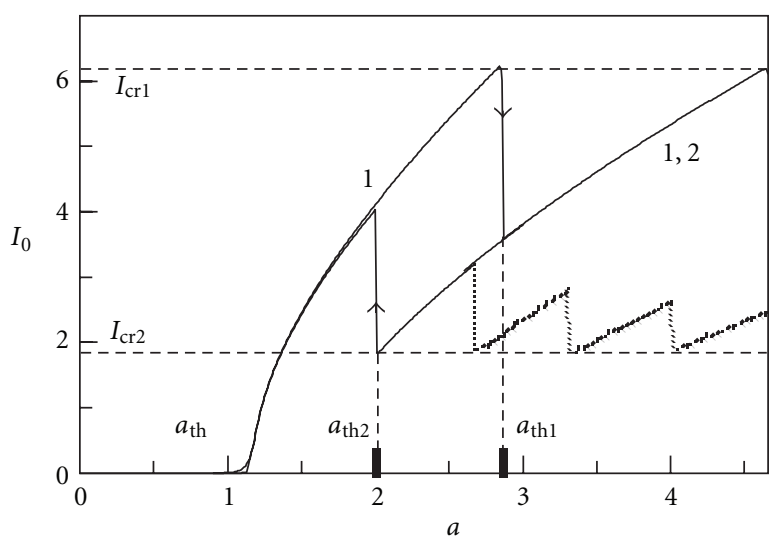

(a)

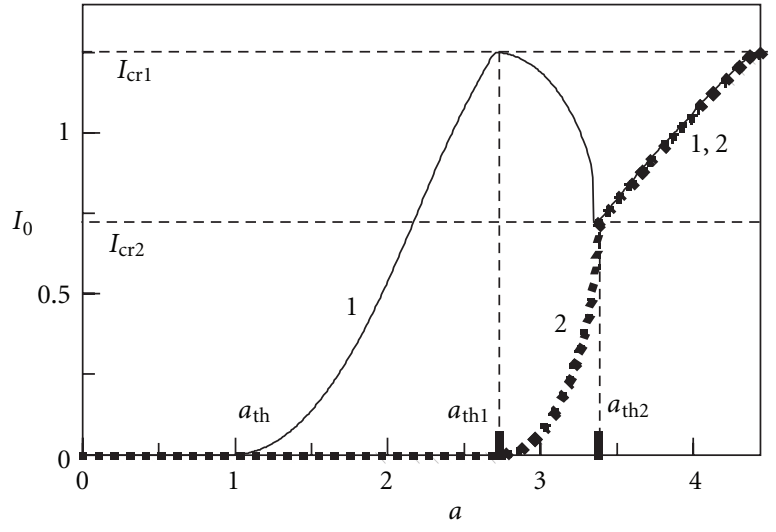

(b)

FIGURE 8: Dependences of the peak intensities $I_{0}$ for two intracavity pulses in established operation on pumping $a$ for hard (a) and soft (b) regimes of excitation and annihilation of the second soliton. The dependences $I_{0}=I_{0}(a)$ for first and second pulses are denoted by digits 1 and 2 , respectively.

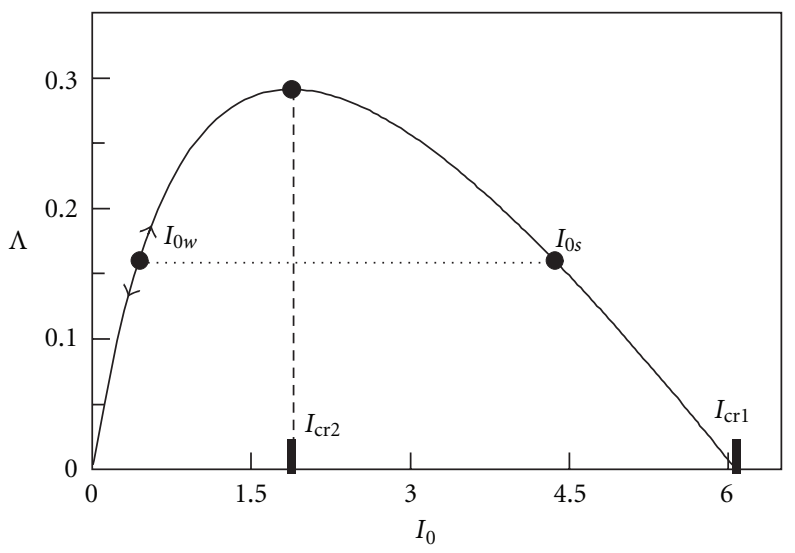

(a)

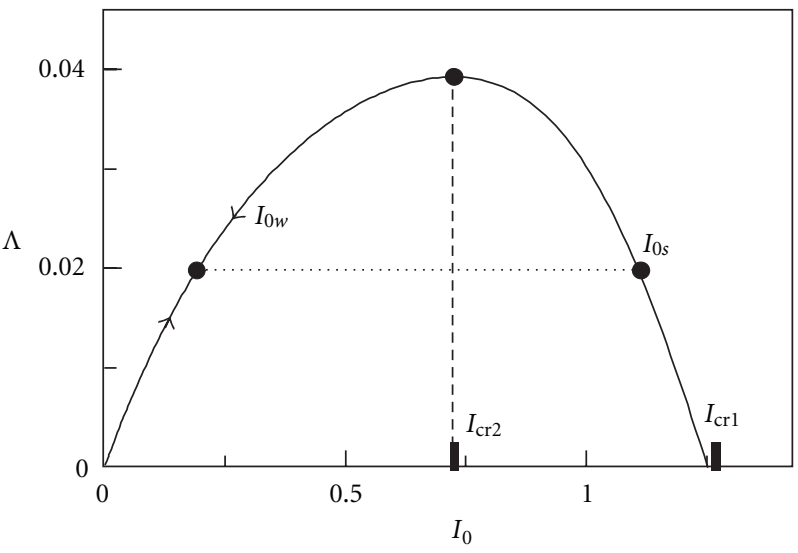

(b)

FIGURE 9: Dependences of the nonlinear-dispersion part of amplification $\Lambda$ for intracavity pulses on their peak intensity $I_{0}$ for hard (a) and soft (b) regimes of excitation and annihilation of the second soliton.

of this dependence, as a rule, the phase difference is close to $\pi / 2$, whereas far from the top (the decreasing part of the dependence), as a rule, the phase difference is close to 0 or $\pi$ (see Section 3).

\section{Bound Steady States of Interacting Solitons}

In the previous section, we have studied phenomena which are related with the interaction of solitons through the gain medium in a condition of fast nonlinear losses and Kerr nonlinearity of refractive index. For these phenomena, the interaction of solitons due to an interference of their wings is insignificant (if the distances between pulses is sufficiently large, then the interference effects are weak). In this section, we study the soliton interaction which is due to an interference of their wings in conditions of the fast complex nonlinearity of intracavity elements.

3.1. Steady States of Pair Bound Solitons. In this section, for our numerical simulation, we have used typical parameters of Er-doped fiber laser with anomalous net dispersion of group velocity. Figure 10 demonstrates the temporal and spectral profiles of a single soliton. Figure 10(a) shows also the change in a phase along the pulse. The soliton has powerful wings which result in large energy bounding pair interacting solitons.

Because of the interaction between solitons, the pair of such solitons is united in the stability formation with a large binding energy_-highly-stable "two soliton molecule." The radiation energy of such molecule is less than the energy of two solitons placed from each other on a long distance. The binding energy for two solitons in this molecule takes the discrete set of values shown on Figure 11. Large binding energies for the low-energy steady states are due to powerful wings of solitons. These steady states are stable.

For the ground steady-state (the first energy level) and for the all odd levels, the field functions are antisymmetric $E_{k}(\tau)=-E_{k}(-\tau)$ if the origin of the coordinate $\tau=0$ corresponds to the point equally spaced from the peaks of the solitons. In this case, the peaks amplitudes of two 


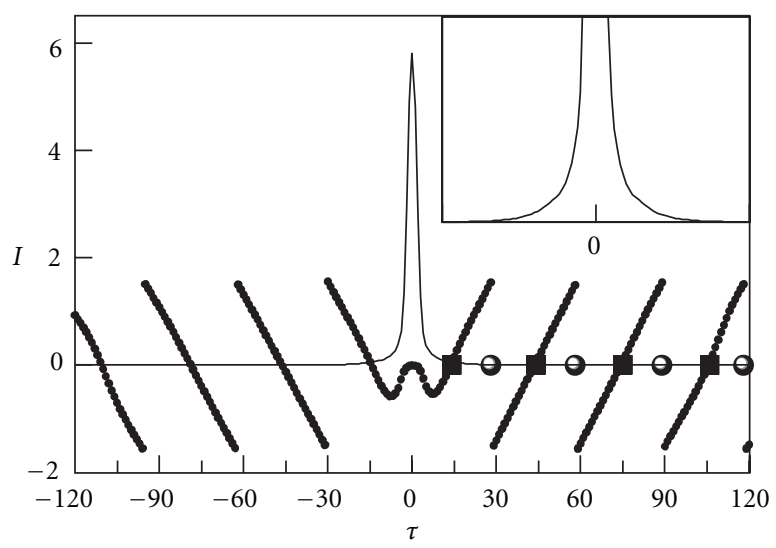

(a)

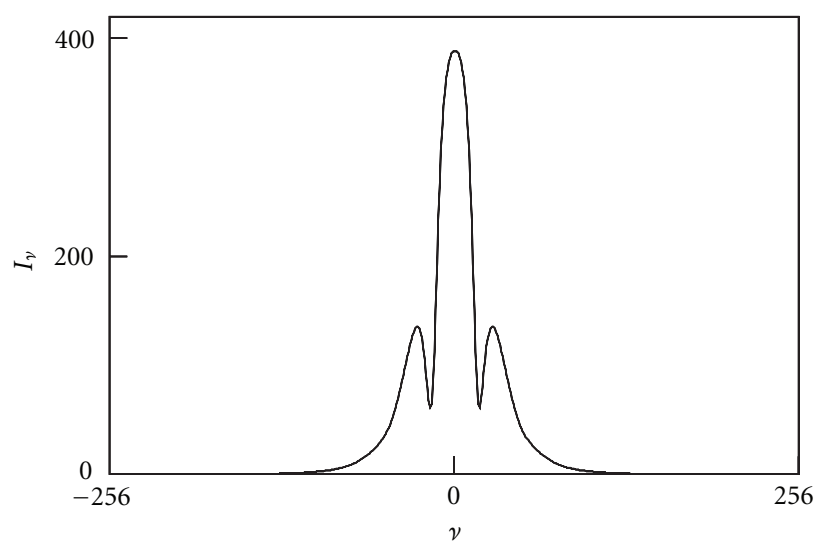

(b)

FIGURE 10: Temporal (a) and spectral (b) distributions of radiation for the single-soliton passive mode-locking. The upper right inset in (a) shows the multiplied soliton pedestal. The change of the phase along the pulse is presented in (a). All phase magnitudes are reduced to the interval $(-\pi,+\pi)$. In the point of the pulse with a maximum amplitude, the phase is equal to zero. The black squares and circles correspond to the points of the pulse where its phase is equal to $\pi$ and 0 , correspondingly.

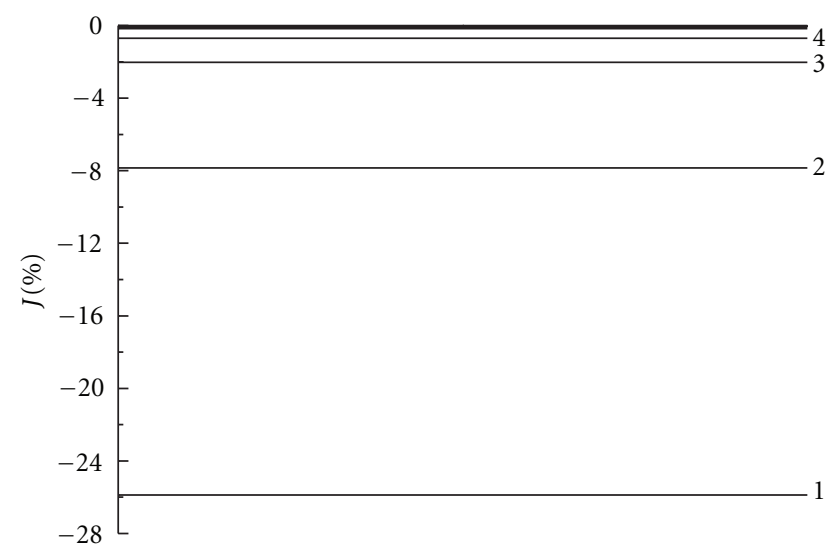

Figure 11: Binding energy of two solitons in stable steady states $J$ expressed in relative units (the binding energy divided by the energy of a single soliton). The laser parameters are the same as in the case of Figure 10.

solitons are in opposite phase $(\delta \varphi \approx \pi)$. For all even steady states, the field functions are symmetric $E_{k}(\tau)=E_{k}(-\tau)$ and the peak amplitudes of two solitons have the same phase $(\delta \varphi \approx 0)$. Figures 12(a), 12(b), and 12(c) show the temporal distributions of intensities in the two-soliton molecule for the cases of the ground steady state (the minimal distance $d_{1}$ between solitons) and for the first and second excited steadystates (the distances between solitons approximately equals the double and triple minimal one $d_{1}$, resp.). In the cases of the ground $(k=1)$ and second excited $(k=3)$ states, the intensity is equal to zero in the center point between solitons. For the first excited state $k=2$, the intensity in this point is distinct from zero. Figures $12(\mathrm{~d}), 12(\mathrm{e})$, and 12(f) show the spectral profiles of the two-soliton molecule. They are symmetric. For odd states, the center spectral component is equal to zero. For even states, it is maximum. For used laser parameters, as indicated above, all steady states of the pair interacting solitons are stable. That is, the two soliton molecule is multistable.

In the bound steady states, the second soliton in the two soliton molecule is arranged in the space point where the first soliton has the phase equal to 0 or $\pi$ (see Figure $10(\mathrm{a})$ ). Under such arrangement, the peak amplitude of one pulse and the wing amplitude of other pulse have the opposite-phase interference. For such interference, the peak intensities of the pulses are decreased and their amplification coefficients become larger (see the dependence $\Lambda=\Lambda\left(\mathrm{I}_{0}\right)$ for $I_{0}>I_{c r 2}$ presented in Figures 7 and 9). We have also obtained the phase difference for the pair pulses equal to $\pi / 2$. In this case, the peak intensity was in vicinity of the maximum of the dependence $\Lambda=\Lambda\left(I_{0}\right)$ ( $I_{0}$ is close to $\left.I_{c r 2}\right)$. As this takes place, for one pulse, the opposite-phase interference is realized, for the other, the in-phase one is done [33].

3.2. Information Sequences of Bound Solitons. One of the usual way of coding the information for its transfer through optical communication fiber lines consists in the following. In equidistant initial sequences of pulses, some pulses are removed. It arises two positions (a pulse is present, and a pulse is absent) which are required for the coding of the information in binary system (zero and unit). Displacement and merge of pulses in such information pulse sequences, that is due to various types of technical perturbations including noise radiation, results in loss of the information. There are various ways of increase of a tolerance to these perturbations. Among them, there is an increase in distance between the neighboring pulses in initial pulse sequence. However, this way results in the decrease in the speed of a transfer of information. In this section, we consider the nonlinear regime of propagation of pulse information sequences. The interaction of neighboring pulses results in the stabilization of this sequence. Because various types of bonds between neighboring pulses can be realized, accordingly, the coding of the information in such sequences can 


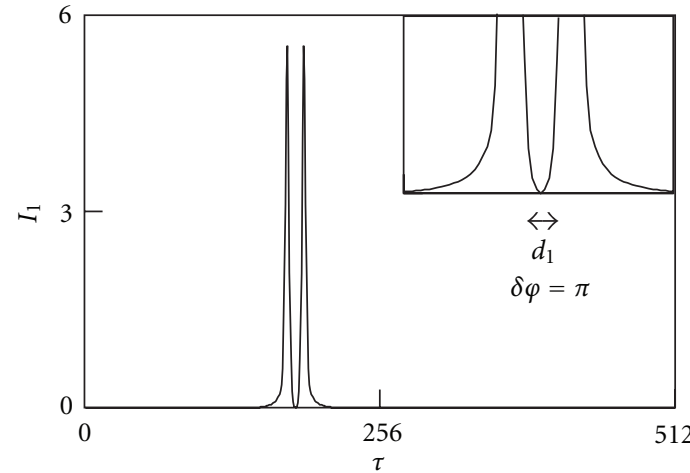

(a)

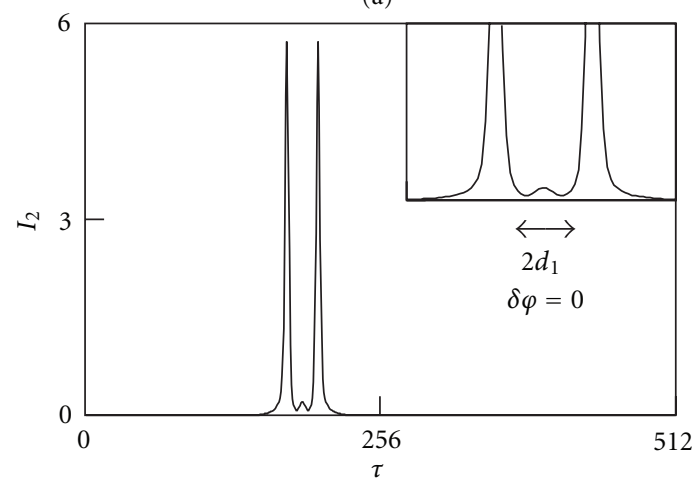

(b)

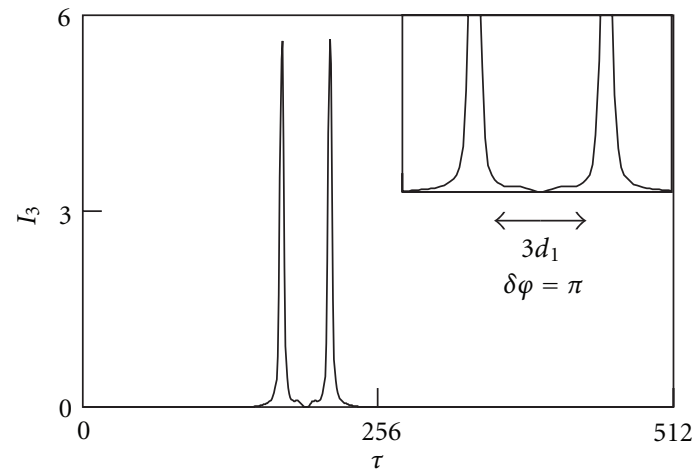

(c)

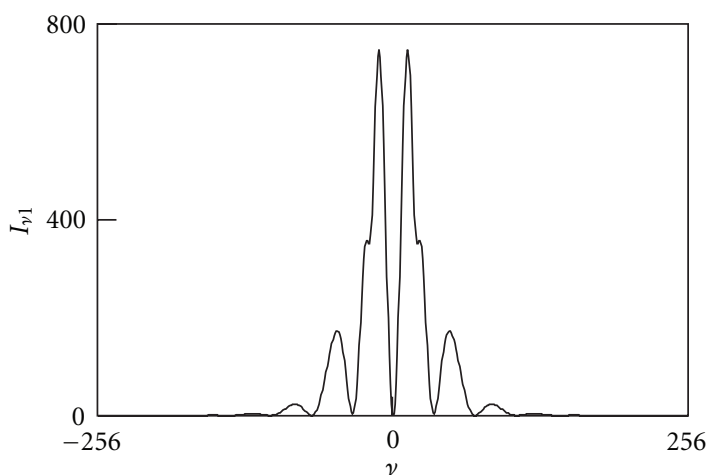

(d)

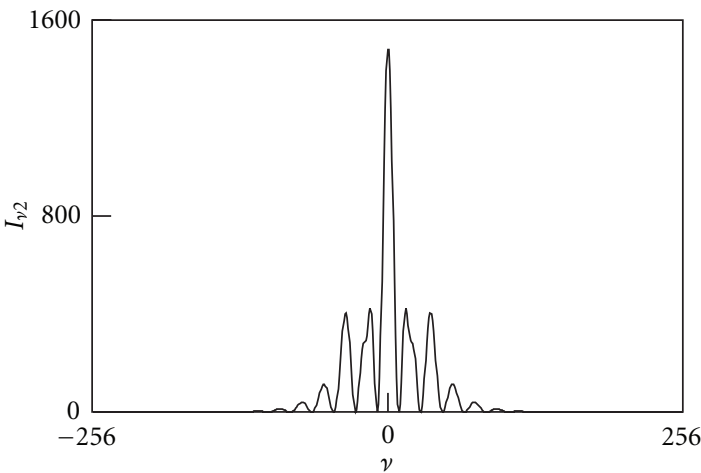

(e)

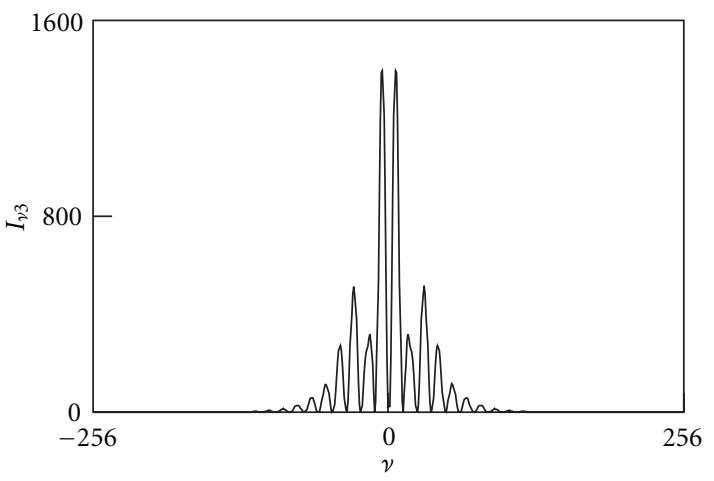

(f)

Figure 12: Temporal and spectral distributions of intensity of the two-soliton molecule (a), (d) for the ground steady state (minimal distance $d_{1}$ between solitons), (b), (e) for the first excited steady state, and (c), (f) for the second excited steady state. All steady states are stable. The laser parameters are the same as in the case of Figure 10.

be realized through various distributions of types of bonds between neighboring pulses along a soliton train. Thanks to powerful wings, the binding energy for such solitons appears high, that provides the high degree of tolerance against various perturbations in the case of such sequences. Dense packing of pulses in such sequence provides high speed of transfer information. Due to large binding energies, such multisoliton molecules are highly stable and noise-proof. Placing several initial pulses on certain distances from each other, after transient process, we have obtained stationary "molecular chains" with any desirable distribution of types of bonds between neighboring solitons along a pulse train. Such sequence is realized more simply with a use of the ground and first excited types of intersoliton bonds for which the binding energies are especially great. Figure 13 shows such information soliton sequence in which the number 28062010 is coded in binary system $(28.06 .2010$ is the data of the Second International Conference: Nonlinear Waves-Theory and Application [41]). Here, the ground type of a bond (smaller distance between pulses) corresponds to unit, and the first excited type of a bond (the greater distance between pulses) corresponds to zero. In binary system, this sequence corresponds to the number 1101011000011000100111010 , that in decimal system is the number 28062010. Really, 1 . $2^{24}+1 \cdot 2^{23}+0 \cdot 2^{22}+\cdots+1 \cdot 2^{1}+0 \cdot 2^{0}=28062010$.

Such soliton trains are highly stable formations. The high stability is primarily due to large binding energies. Furthermore, there exists a second reason of the high stability. 


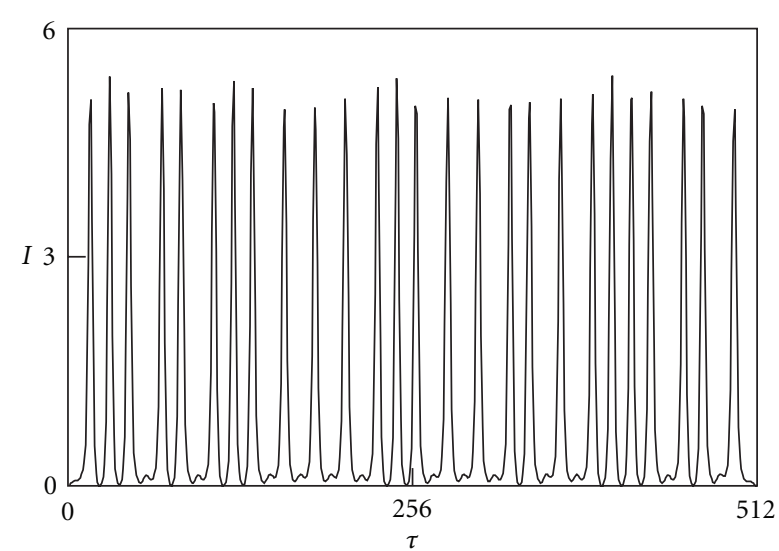

FIGURE 13: Stable molecule chain of bound solitons with the ground and first excited types of bonds in which the number 28062010 is coded in binary system 1101011000011000100111010 . The greater distance between pulses corresponds to zero. The smaller distance between pulses does to unit. The laser parameters are the same as in the case of Figure 10.

It consists in the following. The perturbation energy which was initially localized in the vicinity of some pair of bound solitons is quickly collectivized among all solitons of the train. In the numerical simulation, we have used the random radiation noise to prove this stability. This noise induces up to $10 \%$ fluctuations of peak intensities of solitons but does not change the structure of soliton sequences.

\section{Steady States of Interacting Solitons in Fiber Lasers with Lumped Saturable Absorber}

In the previous sections, we have studied steady states of interacting solitons in passive mode-locking fiber lasers with a nonlinear polarization rotation technique. In this section, we investigate another type of nonlinear losses when passive mode-locking is realized thanks to a saturable absorber. The role of such nonlinear absorber can be played by various materials: carbon nanotubes, graphene, saturable absorbers based on quantum dots, and so on [23-25]. In this case, (2) is replaced by the following one:

$$
E_{n+1}(\tau)=E_{n}(\tau) \exp \left(-\frac{s_{n l}}{1+p I_{n}(\tau)}\right) .
$$

This equation describes the change in the field under its pass through the lumped saturating absorber, where $s_{n l}$ is the losses for a weak field and $p$ is the parameter of a saturation. We have studied the formation of bound states of interacting solitons and obtained analogical results as for the case of nonlinear losses due to the nonlinear polarization rotation technique. Figure 14 shows the distances between two interacting solitons in stable steady states. One can see an alternation of odd and even states as in Figures 11 and 12 in the case of nonlinear losses due to the polarization rotation technique. Here, powerful soliton wings are also realized. These powerful wings result in large bound energies of interacting pulses. For both cases of a realization of nonlinear

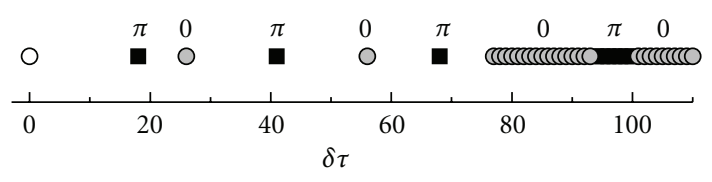

FIGURE 14: Steady-state distances between two solitons interacting through a lumped saturable absorber. The white circle corresponds to the first soliton. The black squares and grey circles do to the steady-state second soliton with the phase differences $\pi$ and 0 , respectively. $a=0.5, D_{r}=0.01, D_{i}=0.1, q=1.5, p=1, s_{n l}=1$.

losses, we have used sufficiently close nonlinear-dispersion parameters of the investigated laser systems.

\section{About Mechanism of Formation of Powerful Soliton Wings}

Large bounding energies of interacting solitons are due to their powerful wings. In this section, we analyze reasons resulting in such wings. Figure 10 (b) demonstrates the additional structure on the bell-shaped spectral profile of a single soliton which has the spectrum sideband form. Sideband generation in soliton spectrum is a well-known phenomenon. The sidebands result from an interference between the soliton and dispersive waves. Such dispersive waves are emitted by a soliton when it circulates in a laser resonator and periodically experiences perturbations caused by the lumped intracavity components. The interference of such wave during several circulations forms the powerful long-distance wings of solitons. This mechanism does not work in the case of a continuously distributed intracavity nonlinear-dispersion medium. In this section, we check the hypothesis about a formation of powerful soliton wings at the expense of dispersive waves.

5.1. Model of a Continuously Distributed Intracavity Nonlinear-Dispersion Medium. In the case of a continuously distributed intracavity nonlinear-dispersion medium the evolution of radiation in a laser cavity is described by the following equation [42]:

$$
\frac{\partial E}{\partial \zeta}=\left(\mathrm{D}_{r}+i \mathrm{D}_{i}\right) \frac{\partial^{2} E}{\partial \tau^{2}}+\left(G-\sigma-\frac{\sigma_{n l}}{1+p|E|^{2}}+i q|E|^{2}\right) E,
$$

where $\sigma$ is linear losses, $G$ is a saturable amplification (see (3)), and the term $\sigma_{n l} /\left(1+p|E|^{2}\right)$ describes the continuously distributed variant of nonlinear losses (4). In the case of $\mathrm{D}_{i}=0, q=0$, this equation transforms into the nonlinear diffusion equation [43] which describes passive modelocking of lasers when any phase-modulation effects may be ignored. Equation (5) was obtained as the generalization of this nonlinear diffusion equation with a purpose of an analysis of effects of phase modulation of ultrashort pulses in passive mode-locked lasers. In the frame of (5), the multiplepulse operation and the multihysteresis phenomena (see 
Figure 3) were investigated in the papers $[44,45]$. In the case $p I \ll 1$, (5) has the form

$$
\frac{\partial E}{\partial \zeta}=\left(\mathrm{D}_{r}+i \mathrm{D}_{i}\right) \frac{\partial^{2} E}{\partial \tau^{2}}+\left(G-\sigma_{0}+(r+i q)|E|^{2}\right) E,
$$

where $\sigma_{0}=\sigma+\sigma_{n l}$ is the total linear losses, $r=p \sigma_{n l}$. It is the simplest equation taking into account a frequency dispersion of gain-losses and a refractive index, a nonlinearity of losses and a refractive index, and also a saturation of an amplification. Equation (6) describes the two lasing regimes: an operation with radiation filling totally laser resonator and a regime with single ultrashort pulse in laser cavity. The amplitude of this single pulse in the case of stationary generation is described by the expression

$$
E=E_{0} \frac{\exp (i \Omega \tau-i \delta k \zeta)}{\cosh ^{1+i \alpha} \beta \tau}
$$

where the peak amplitude of a pulse $E_{0}$, its reverse duration $\beta$, its frequency chirp $\alpha$, parameters $\Omega$ and $\delta k$ are determined from a system of algebraic equations [42]. The spectral profile of the ultrashort pulse (7) is determined by the following analytical expression $[42,46]$ :

$$
I_{\nu}=\frac{\pi^{2}\left|E_{0}\right|^{2}}{\beta^{2}} \frac{\sinh \pi \alpha}{\alpha(\cosh \pi \alpha+\cosh (\pi \nu / \beta))},
$$

where $v$ is a frequency detuning from the center frequency of soliton radiation. With increasing chirp $\alpha$, the spectral profile is changed from a bell-shaped form to a rectangle one (see Figure 15(a)). Figure 15(b) shows the change in the frequency chirp $\alpha$ on the plate of the nonlinear-dispersion parameters $\xi=q / r, \theta=D_{i} / D_{r}$. Equation (6) has also a solution with indefinitely increasing amplitude $E \rightarrow \infty$ (that is $J \rightarrow \infty$ ) which is not correct because of breakdown of the condition $p I \ll 1$.

Equation (6) is close to the following equation:

$$
\frac{\partial \Psi}{\partial t}=\left(\mathrm{d}_{r}+i \mathrm{~d}_{i}\right) \frac{\partial^{2} \Psi}{\partial z^{2}}+\left[g+\left(c_{1}+i c_{2}\right)|\Psi|^{2}\right] \Psi
$$

where $d_{r}, d_{i}, g, c_{1}, c_{2}$ are constant parameters. This equation is used in a description of hydrodynamical phenomena $[47,48]$ (see also $[49,50]$ ). It has also the solution in the form (7) $[48,49,51]$. However, there exists the principle distinction between these equations: equation (6) has the stabile solution in the form of a single stationary soliton described by (7), but in the case of (9), such stationary solution is always unstable. In the case of (6), the stabilization of the single-pulse solution is realized through a saturation of the amplification $G$. If the parameter of the gain saturation is equal to zero $b=0$ (see (3)), then (6) is transformed into (9). The stabilization of a single pulse can be achieved at the expense of nonlinear losses described by additional highorder nonlinearities $[32,52]$. However, in this case, the exact elegant analytical solutions (7) and (8) for an amplitude and a spectrum of a single stationary pulse do not hold.
5.2. Model with Combination of Lumped and Continuously Distributed Nonlinear Losses. We study passive mode-locked laser with the combination of the continuously distributed saturable absorber included in (5) and the lumped saturable absorber described by (4). To follow the change of properties of soliton wings due to dispersive waves and correspondingly of properties of steady states of a pair of interacting solitons, we decrease the magnitude of the lumped saturable absorber $s_{n l}$ up to zero. Simultaneously we increase the value of the distributed nonlinear losses $\sigma_{n l}$ thus that the total losses for a weak signal for one pass of a field through the resonator remain constant. If our hypothesis is true, then dispersive waves should weaken up to zero and the amplitude of soliton wings should decrease that will result in the change of properties of bound steady states.

Figure 16 shows the spectral change in a single soliton with varying lumped part of nonlinear losses $s_{n l}$. One can see the decrease and disappearance of sidebands in the soliton spectrum with decreasing $s_{n l}$. Figure 17 shows the decrease of a bounding energy for the first excited steady state of a pair of bounding solitons with decreasing values $s_{n l}$. Figure 18 shows the decrease of the soliton wing with decreasing lumped part of nonlinear losses. These results demonstrate the role of dispersive waves in a formation of powerful soliton wings which determine properties of bound steady state of interacting solitons.

\section{Discussion}

We have developed a theoretical model to describe several behaviors which are usually observed in passively modelocked fiber lasers. The model is based on a fiber exhibiting optical Kerr nonlinearity, gain and group velocity dispersions, and saturable gain. For our analysis, we use typical parameters for ytterbium- and erbium-doped fiber lasers operating in the normal and anomalous dispersion regimes. Two mechanisms of nonlinear losses have been analyzed. One of them is related with the nonlinear polarization rotation technique. The other is due to a saturable absorber. For both mechanisms, the analogical results have been obtained. In our study, we have chosen such laser parameters for which the researched phenomena are manifested most clearly. With other parameters, we have observed various modifications of investigated regimes. For example, we have observed the temporal oscillations of soliton amplitudes in bound states. For some parameters, the lower states were unstable. As a result of this instability, a soliton pair transits into a higher steady state. With other parameters, this instability results in a merge of solitons. With certain parameters, the investigated phenomena are masked by period-doubling effects.

The multiple-pulse passive mode-locking has been carefully investigated. It is determined by a coexistence and a competition of pulses amplified in a common gain medium. The multiple-pulse operation is realized through the effect of a quantization of intracavity radiation into identical dissipative solitons. A number of identical pulses in a laser cavity as a function of pumping are determined by multihysteresis dependence. These results are in good agreement with 


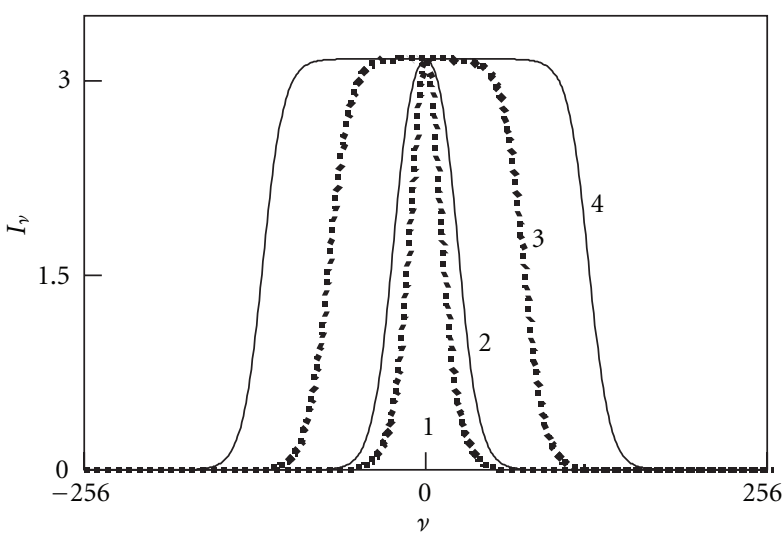

(a)

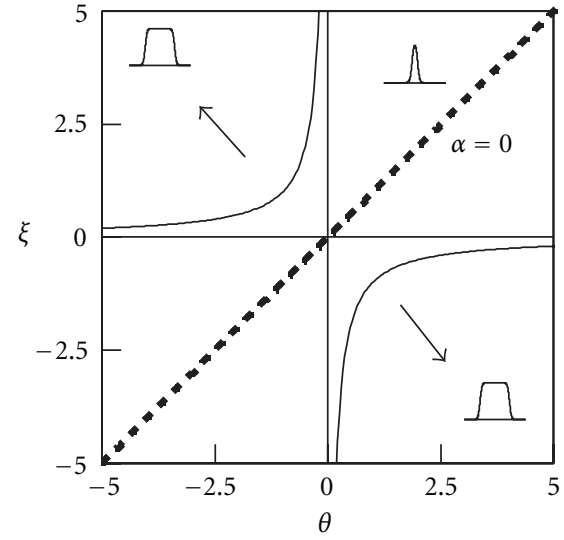

(b)

FIGURE 15: Spectra of ultrashort pulse described by (8). (a) Spectral profiles of established solitons with different chirps: (1) $\alpha=0$, (2) $\alpha=1$, (3) $\alpha=3$, (4) $\alpha=5$. (b) Variation of the chirp $\alpha$ on the plane of the nonlinear-dispersion parameters $(\xi, \theta)$. For the dashed line $\alpha=0$, for the solid curves $\alpha= \pm \sqrt{2}$, the arrows point the directions of maximal increase in the chirp $\alpha$. The spectral profiles in the figure (b) demonstrate typical spectra for the three areas separated from each other by the solid curves.

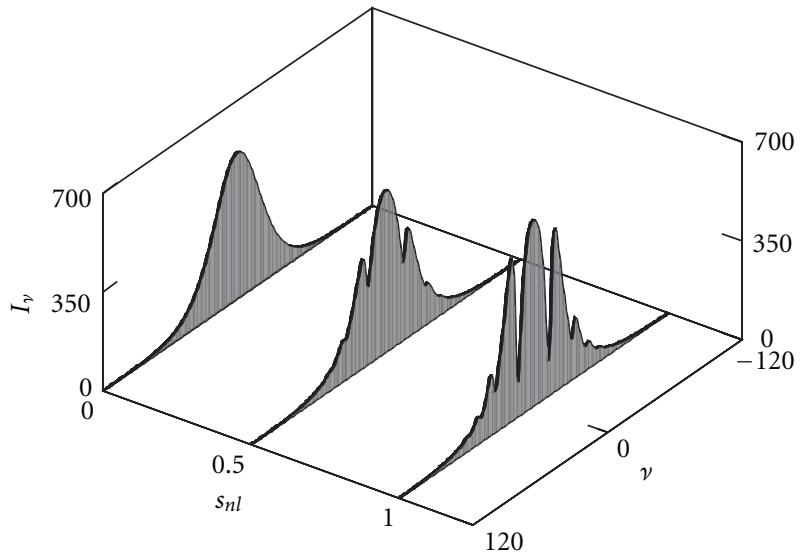

Figure 16: Change in spectrum of single ultrashort pulse with the change in a value of lumped part of saturable absorber $s_{n l}$. The total value of nonlinear losses remains the same.

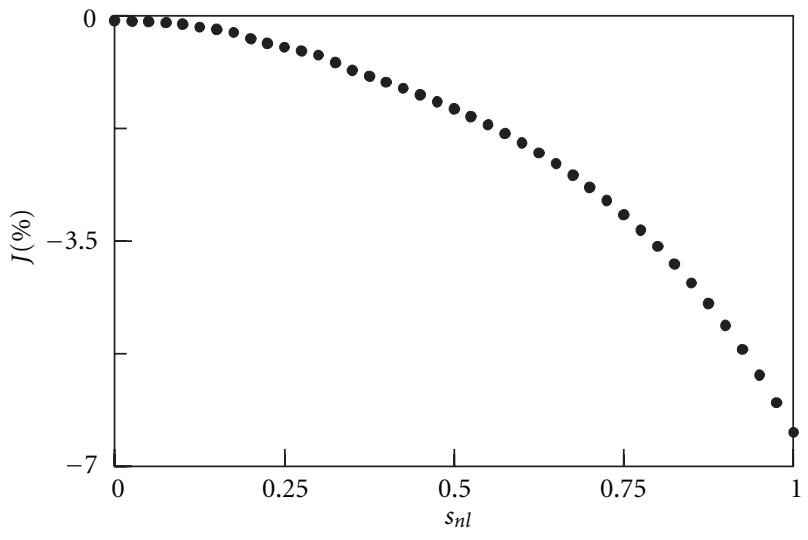

Figure 17: Bound energy for the first excited steady state with changing value of a lumped part of a saturable absorber $s_{n l}$.

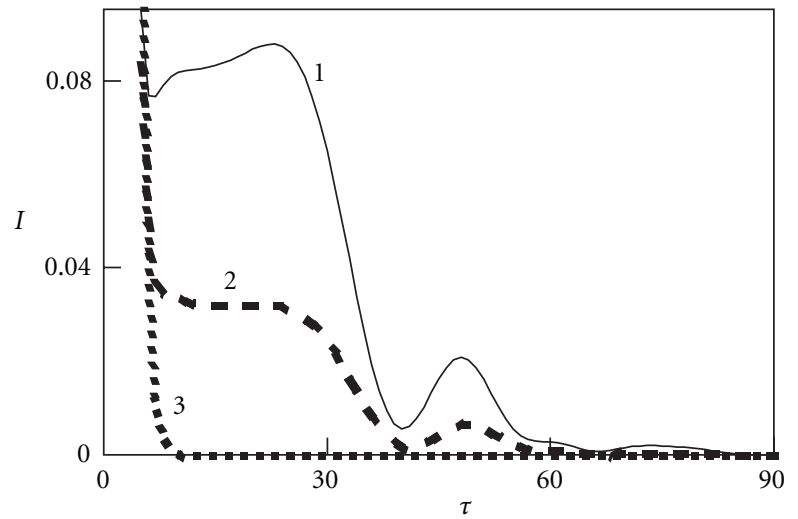

FIGURE 18: Change in the intensity of single-soliton wing with the change in a value of lumped part of saturable absorber $s_{n l}$ : (1) $s_{n l}=$ 1 , (2) $s_{n l}=0.75$, (3) $s_{n l}=0$. The total value of nonlinear losses including lumped and distributed parts remains the same.

experimental data reported in the literature (see e.g., [17]). It is shown that the multihysteresis dependence of a number of pulses on pumping results in analogical multihysteresis dependences for an intracavity energy and a peak intensity of intracavity solitons.

It is found that a competition and a coexistence of solitons in a laser cavity is determined by the dependence of the nonlinear-dispersion part of an amplification on a peak intensity of solitons $\Lambda=\Lambda\left(I_{0}\right)$ (see Figures 7 and 9). For achievement of a single-pulse generation, it is necessary to increase the parameter $I_{c r 2}$ that the condition $I_{0}<I_{c r 2}$ is satisfied for all intracavity pulses. In this case, the multiplepulse generation is suppressed and, as result, the single pulse operation is realized. In such a way, it is possible to expect 
a generation of pulses with extremely high energy. This problem demands detailed studying.

Usually at a multiple-pulse generation, all pulses have identical parameters. It is realized when a hard regime of an annihilation and an excitation of new pulses is realized. However, as it is shown in the given paper, the soft regime of an annihilation and an excitation is also possible. In this case, one of pulses has smaller amplitude than other pulses having identical parameters.

An interaction of pulses through an interference of their wings in fast intracavity nonlinear medium is more fine effect than the realization of a multiple-pulse generation. The dependence of the nonlinear-dispersion part of an amplification on a peak intensity of solitons $\Lambda=\Lambda\left(I_{0}\right)$ dictates the phase difference of interacting solitons: it is close to $\pi, \pi / 2,0$. However, various stationary states have distinguished degrees of stability. The degree of stability essentially depends on type of nonlinearity and from values of nonlinear-dispersion parameters. This fact is well known from the papers [32, 52]. If master equation is close to nonlinear Schrödinger equation (dissipative terms are small), then all steady states are unstable, but steady states with $\pi / 2$ phase difference have extremely weak instability, that is, they are practically stable [52]. If dissipative terms are not small, then $\pi / 2$ steady states are merely stable [32] (authors of both the papers used model with complex quadratic dispersion and complex cubic-quintic nonlinearity).

Thanks to powerful wings, large binding energies of interacting solitons can be obtained. In our numerical experiment, we have realized high-stable noise-proof multisoliton molecules. We have shown that sets of various types of bonds between neighboring pulses in such molecule can be obtained. Accordingly, the coding of the information in these soliton sequences can be realized through various distributions of types of bonds between neighboring pulses along a soliton chain. Dense packing of pulses in the bound soliton sequence provides high speed of transfer of an information in fiber communications line working in nonlinear bound-soliton-based regime. Such systems can be also useful for storage and processing of an information. The problem of such coding of a data stream into a bound soliton chain (and also of decoding) demands a detailed study.

In the case of a realization of the same type of a bond between all neighboring solitons in a structure with a very large number of pulses, we obtain an analog of a crystal of finite extent. This fully ordered state was named a soliton crystal by analogy with the results of Mitschke and his coauthors on pulse reshaping in a passive synchronously pumped fiber-ring resonator [53, 54]. If such soliton crystal with a single type of a bond fills completely the total ring laser cavity, then harmonic passive mode-locking is realized. This regime is interesting for a creation of generators of ultrashort pulses with superhigh rate of a repetition of pulses in output radiation. Generators of pair of ultrashort pulses with the certain fixed distance between pulses also can find numerous applications.

We have investigated a mechanism of a formation of powerful soliton wings connected with a lumped saturable absorber. In case of the nonlinear polarization rotation technique, the nonlinear losses are realized in the polarizer and they are lumped. In the case of a saturable absorber, the nonlinear losses can be lumped and continuously distributed. We have analyzed a laser model with a combination of lumped and continuously distributed parts of the total saturable absorber. Keeping the total nonlinear losses constant, we have varied their lumped part and studied properties of bound steady states of interacting solitons. We have found that the dispersive waves emitted by solitons because of lumped intracavity elements form powerful soliton wings resulting in great bounding energy of interacting solitons in steady states. The role of dispersive waves in a realization of a mechanism of a repulse between solitons and, as a result, realization of harmonic passive mode-locking calls for further investigation.

\section{Conclusion}

On basis of numerical simulation, we have studied the basic features in a realization of multiple-pulse operation of passive mode-locked fiber lasers. It is found that in the case of the hard regime of an annihilation and an excitation of new solitons with changing pump, all intracavity solitons have identical parameters (a peak intensity, a duration, a chirp, and so on). However, in the case of the soft regime of an annihilation and an excitation, one of pulses has a peak intensity smaller than other identical intracavity solitons. It is found that the multihysteresis dependence of a number of pulses on pump results in an analogical multihysteresis dependence for the intracavity radiation energy and for the peak intensity of identical solitons. An interaction of pulses through an interference of their wings in fast intracavity nonlinear medium has been investigated. Bound steady states of a two soliton molecule are determined. We have demonstrated the possibility to form information soliton sequences with any desirable distribution of the types of bonds between neighboring pulses along soliton trains. Thanks to large values of binding energies, such sequences have a high level of stability against perturbations. It is found that dispersive waves emitted by solitons because of lumped nonlinear losses form powerful soliton wings resulting in great bounding energy of interacting solitons in steady states.

\section{Acknowledgments}

The work is supported by the Agence Nationale de la Recherche (Contract ANR-2010-BLANC-0417-01SOLICRISTAL) and by the RF President's Grant MK2602.2011.2.

\section{References}

[1] P. G. Kryukov, "Ultrashort-pulse lasers," Quantum Electronics, vol. 31, no. 2, pp. 95-119, 2001.

[2] A. A. Ivanov, M. V. Alfimov, and A. M. Zheltikov, "Femtosecond pulses in nanophotonics," Physics-Uspekhi, vol. 47, no. 7, pp. 687-704, 2004. 
[3] M. E. Fermann and I. Hartl, "Ultrafast fiber laser technology," IEEE Journal on Selected Topics in Quantum Electronics, vol. 15, no. 1, Article ID 4773318, pp. 191-206, 2009.

[4] T. Brabec and F. Krausz, "Intense few-cycle laser fields: frontiers of nonlinear optics," Reviews of Modern Physics, vol. 72, no. 2, pp. 545-591, 2000.

[5] E. V. Baklanov and P. V. Pokasov, "Optical frequency standards and femtosecond lasers," Quantum Electronics, vol. 33, no. 5, pp. 383-400, 2003.

[6] G. P. Agrawal, Nonlinear Fiber Optics, Elsevier Science and Technology Books, Academ Press, Burlington, Mass, USA, 2006.

[7] N. Akhmediev and A. Ankiewicz, Dissipative Solitons, Lecture Notes in Physics, Springer, Berlin, Germany, 2005.

[8] F. W. Wise, A. Chong, and W. H. Renninger, "High-energy femtosecond fiber lasers based on pulse propagation at normal dispersion," Laser \& Photonics Reviews, vol. 2, no. 1-2, pp. 5873, 2008.

[9] S. Chouli and P. Grelu, "Rains of solitons in a fiber laser," Optics Express, vol. 17, no. 14, pp. 11776-11781, 2009.

[10] D. Y. Tang, L. M. Zhao, X. Wu, and H. Zhang, "Soliton modulation instability in fiber lasers," Physical Review A, vol. 80, pp. 023806-023813, 2009.

[11] F. Amrani, A. Haboucha, M. Salhi, H. Leblond, A. Komarov, and F. Sanchez, "Dissipative solitons compounds in a fiber laser. Analogy with the states of the matter," Applied Physics $B$, vol. 99, no. 1-2, pp. 107-114, 2010.

[12] S. Kobtsev, S. Kukarin, S. Smirnov, S. Turitsyn, and A. Latkin, "Generation of double-scale femto/pico-second optical lumps in mode-locked fiber lasers," Optics Express, vol. 17, no. 23, pp. 20707-20713, 2009.

[13] P. Grelu, F. Belhache, F. Gutty, and J. M. Soto-Crespo, "Relative phase locking of pulses in a passively mode-locked fiber laser," Journal of the Optical Society of America B, vol. 20, no. 5, pp. 863-870, 2003.

[14] H. A. Haus, “Mode-locking of lasers," IEEE Journal on Selected Topics in Quantum Electronics, vol. 6, no. 6, pp. 1173-1185, 2000.

[15] V. I. Denisov, B. N. Nyushkov, and V. S. Pivtsov, "Self-modelocked all-fibre erbium laser with a low repetition rate and high pulse energy," Quantum Electronics, vol. 40, no. 1, pp. 2527, 2010.

[16] A. Komarov, A. Haboucha, K. Komarov, H. Leblond, M. Salhi, and F. Sanchez, "Soliton interaction in fiber laser," in Recent Research Developments in Optics, S. G. Pandalai, Ed., vol. 7, pp. 63-112, 2009.

[17] D. Y. Tang, W. S. Man, and H. Y. Tam, "Stimulated soliton pulse formation and its mechanism in a passively mode-locked fibre soliton laser," Optics Communications, vol. 165, no. 4, pp. 189-194, 1999.

[18] A. Hideur, T. Chartier, M. Sahli, C. Ozkul, C. M. Brunel, and F. Sanchez, "Mode-lock, Q-switch and CW operation of an Ybdoped double-clad fiber ring laser," Optics Communications, vol. 198, no. 1, pp. 141-146, 2001.

[19] A. B. Grudinin, D. J. Richardson, and D. N. Payne, "Energy quantisation in figure eight fibre laser," Electronics Letters, vol. 28, no. 1, pp. 67-68, 1992.

[20] A. Komarov, H. Leblond, and F. Sanchez, "Multistability and hysteresis phenomena in passively mode-locked fiber lasers," Physical Review A, vol. 71, no. 5, Article ID 053809, pp. 1-9, 2005.
[21] D. Y. Tang, L. M. Zhao, B. Zhao, and A. Q. Liu, "Mechanism of multisoliton formation and soliton energy quantization in passively mode-locked fiber lasers," Physical Review A, vol. 72, no. 4, pp. 1-9, 2005.

[22] A. Komarov, H. Leblond, and F. Sanchez, "Theoretical analysis of the operating regime of a passively-mode-locked fiber laser through nonlinear polarization rotation," Physical Review A, vol. 72, no. 6, pp. 063811-063818, 2005.

[23] J.-C. Chiu, Y.-F. Lan, and J.-J. Kang, "Passivelly mode-locked lasers using saturable absorber incorporating dispersed single wall carbon nanotudes," in Proceedings of the IEEE Electronic Component and Technology Conference, pp. 827-830, San Diego, Calif, USA, 2009.

[24] H. Zhang, D. Tang, R. J. Knize, L. Zhao, Q. Bao, and K. P. Loh, "Graphene mode locked, wavelength-tunable, dissipative soliton fiber laser," Applied Physics Letters, vol. 96, no. 11, Article ID 111112, 2010.

[25] Z. Sun, T. Hasan, D. Popa et al., "Ultrafast fiber laser modelocked by graphene based saturable absorber," in Proceedings of the Lasers and Electro-Optics/Quantum Electronics and Laser Science Conference: 2010 Laser Science to Photonic Applications (CLEO/QELS 2010), San Jose, Calif, USA, May 2010.

[26] P. Grelu, F. Belhache, F. Gutty, and J. M. Soto-Crespo, "Phaselocked soliton pairs in a stretched-pulse fiber laser," Optics Letters, vol. 27, no. 11, pp. 966-968, 2002.

[27] M. Olivier, V. Roy, M. Piché, and F. Babin, "Pulse collisions in the stretched-pulse fiber laser," Optics Letters, vol. 29, no. 13, pp. 1461-1463, 2004.

[28] D. Y. Tang, W. S. Man, H. Y. Tam, and P. D. Drummond, "Observation of bound states of solitons in a passively modelocked fiber laser," Physical Review A, vol. 64, no. 3, Article ID 033814, 2001.

[29] A. K. Komarov, K. P. Komarov, H. Leblond, and F. Sanchez, "Spectral control over the interaction of ultrashort pulses in fiber lasers," Optics and Spectroscopy, vol. 103, no. 5, pp. 825830, 2007.

[30] N. Akhmediev, J. M. Soto-Crespo, M. Grapinet, and P. Grelu, "Dissipative soliton interactions inside a fiber laser cavity," Optical Fiber Technology, vol. 11, no. 3, pp. 209-228, 2005.

[31] B. A. Malomed, "Bound solitons in the nonlinear SchrödingerGinzburg-Landau equation,” Physical Review A, vol. 44, no. 10, pp. 6954-6957, 1991.

[32] N. Akhmediev, A. Ankiewicz, and J. M. Soto-Crespo, "Multisoliton solutions of the complex ginzburg-landau equation," Physical Review Letters, vol. 79, no. 21, pp. 4047-4051, 1997.

[33] A. Komarov, K. Komarov, and F. Sanchez, "Quantization of binding energy of structural solitons in passive mode-locked fiber lasers," Physical Review A, vol. 79, no. 3, Article ID 033807, 2009.

[34] K. S. Abedin, J. T. Gopinath, L. A. Jiang, M. E. Grein, H. A. Haus, and E. P. Ippen, "Self-stabilized passive, harmonically mode-locked stretched-pulse erbium fiber ring laser," Optics Letters, vol. 27, no. 20, pp. 1758-1760, 2002.

[35] C. X. Yu, H. A. Haus, E. P. Ippen, W. S. Wong, and A. Sysoliatin, "Gigahertz-repetition-rate mode-locked fiber laser for continuum generation," Optics Letters, vol. 25, no. 19, pp. 1418-1420, 2000.

[36] B. Ortaç, A. Hideur, G. Martel, and M. Brunel, "2-GHz passive harmonically mode-locked Yb-doped double-clad fiber laser," Applied Physics B, vol. 81, no. 4, pp. 507-509, 2005.

[37] A. Komarov, H. Leblond, and F. Sanchez, "Passive harmonic mode-locking in a fiber laser with nonlinear polarization rotation," Optics Communications, vol. 267, no. 1, pp. 162-169, 2006. 
[38] A. Komarov, A. Haboucha, and F. Sanchez, "Ultrahighrepetition-rate bound-soliton harmonic passive mode-locked fiber lasers," Optics Letters, vol. 33, no. 19, pp. 2254-2256, 2008.

[39] A. Haboucha, H. Leblond, M. Salhi, A. Komarov, and F. Sanchez, "Coherent soliton pattern formation in a fiber laser," Optics Letters, vol. 33, no. 5, pp. 524-526, 2008.

[40] Y. I. Khanin, Principles of Laser Dynamics, Elsevier, Amsterdam, The Netherlands, 1995.

[41] A. Komarov, K. Komarov, D. Meshcheriakov, F. Amrani, and F. Sanchez, "Dissipative solitons in passive mode-locked fiber lasers with nonlinear polarization rotation technique," in Proceedings of the Second International Conference:Nonlinear Waves-Theory and Applications, Technical Digest, p. 207, Beijing, China, June 2010.

[42] K. P. Komarov, "Theory of stationary ultrashort pulses in solid-state lasers with passive mode-locking," Optics and Spectroscopy, vol. 60, no. 2, pp. 231-234, 1986.

[43] K. P. Komarov and V. D. Ugozhaev, "Steady-state pulses in solid-state lasers with passive mode locking," Optics and Spectroscopy, vol. 55, no. 5, pp. 564-568, 1983.

[44] A. K. Komarov, K. P. Komarov, and A. S. Kuch'yanov, "On phase-modulational bifurcation during passive mode locking in lasers," JETP Letters, vol. 67, no. 4, pp. 280-283, 1998.

[45] A. K. Komarov and K. P. Komarov, "Multistability and hysteresis phenomena in passive mode-locked lasers," Physical Review E, vol. 62, no. 6 B, pp. R7607-R7610, 2000.

[46] K. P. Komarov, A. S. Kuch'yanov, and V. D. Ugozhayev, "Generation of stationary ultra-short pulses by a passive mode-locking solid-state laser," Optics Communications, vol. 57, no. 4, pp. 279-284, 1986.

[47] L. M. Hocking, K. Stewartson, J. T. Stuart, and S. N. Brown, "A nonlinear instability burst in plane parallel flow," Journal of Fluid Mechanics, vol. 51, no. 4, pp. 705-735, 1972.

[48] L. M. Hocking and K. Stewartson, "On the nonlinear response of a marginally unstable plane parallel flow to a twodimensional disturbance," Proceedings of the Royal Society A, vol. 326, pp. 289-313, 1972.

[49] A. C. Newell, "Envelope equations," Lectures in Applied Mathematics, vol. 15, pp. 157-163, 1974.

[50] A. C. Newell and J. A. Whitehead, "Finite bandwidth, finite amplitude convection," Journal of Fluid Mechanics, vol. 38, no. 2, pp. 279-303, 1969.

[51] N. R. Pereira and L. Stenflo, "Nonlinear Schrödinger equation including growth and damping," Physics of Fluids, vol. 20, no. 10, pp. 1733-1734, 1977.

[52] V. V. Afanasjev, B. A. Malomed, and P. L. Chu, "Stability of Bound States of pulses in the Ginzburg-Landau equations," Physical Review E, vol. 56, no. 5, pp. 6020-6023, 1997.

[53] B. A. Malomed, A. Schwache, and F. Mitschke, "Soliton lattice and gas in passive fiber-ring resonators," Fiber and Integrated Optics, vol. 17, no. 4, pp. 267-277, 1998.

[54] S. Rutz and F. Mitschke, "Towards thermodynamics of solitons: cooling," Journal of Optics B, vol. 2, no. 3, pp. 364-366, 2000. 

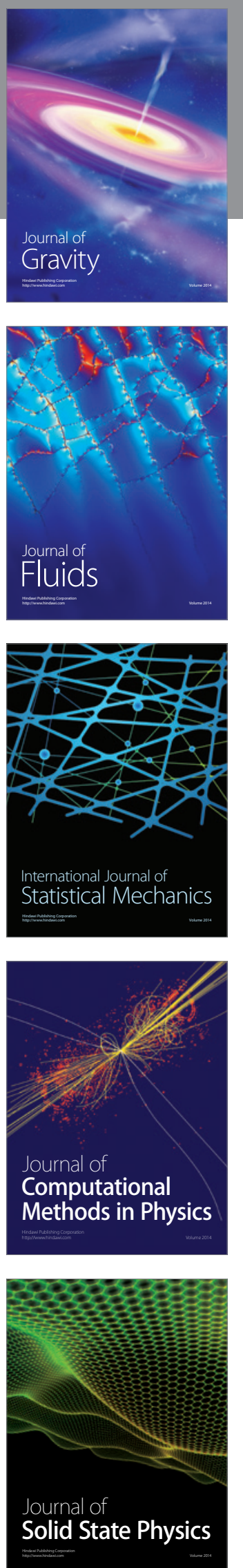

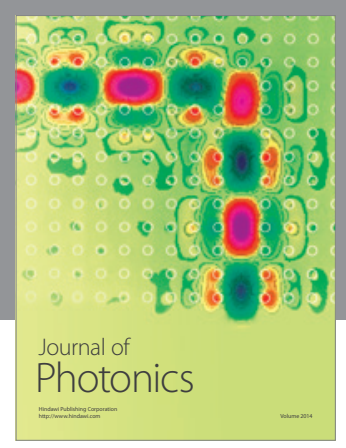

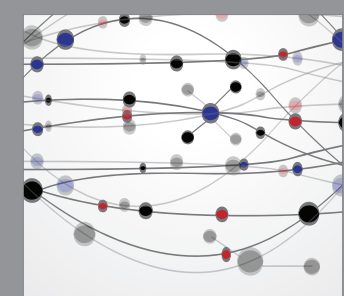

The Scientific World Journal
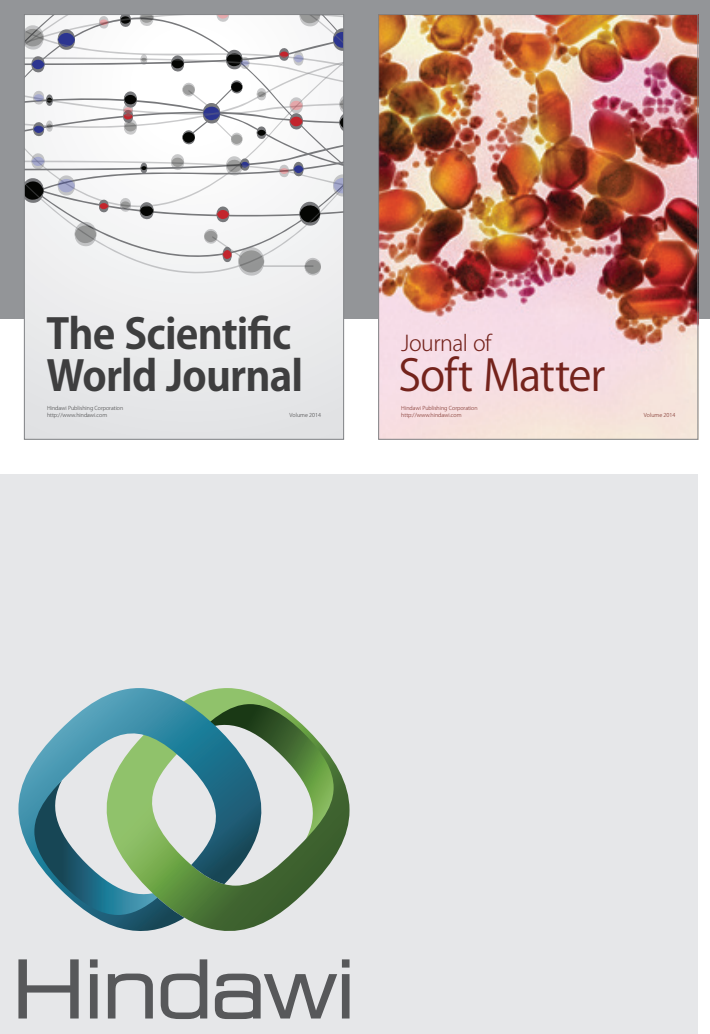

Submit your manuscripts at

http://www.hindawi.com
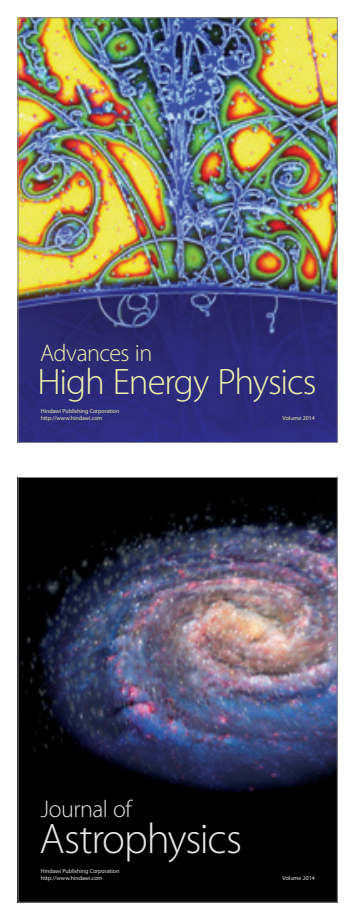
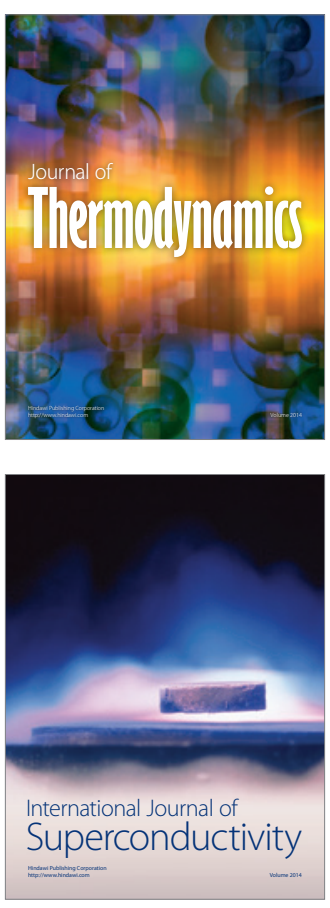
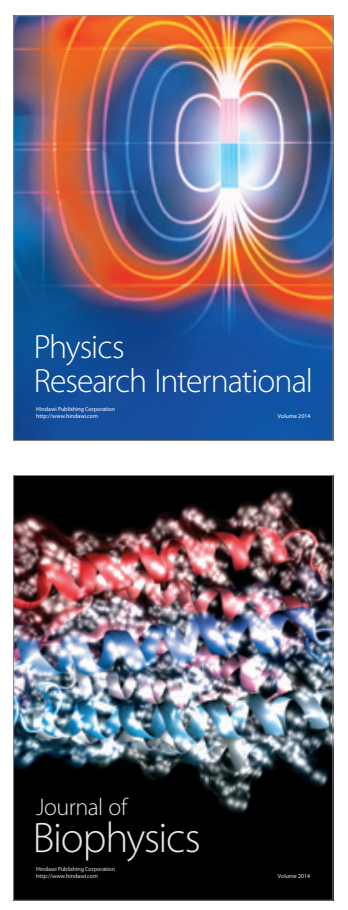
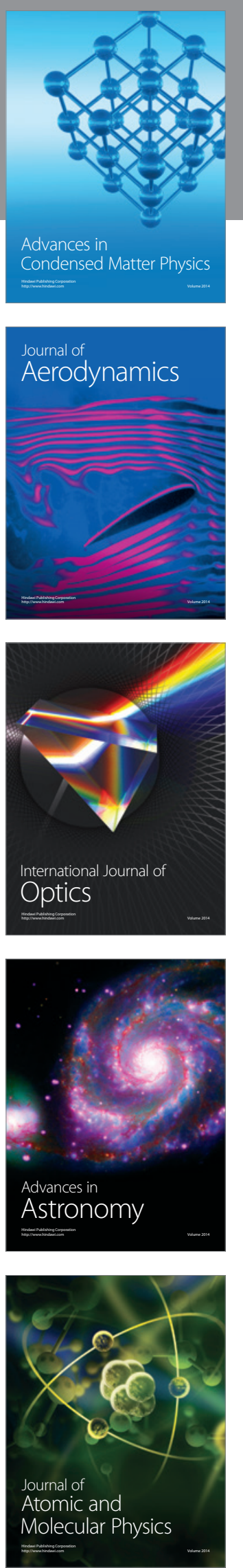